progress was excellent and she remains well one year later.

\section{Discussion}

Our case illustrates the persistence of severe rheumatic carditis and emphasises a number of points in the diagnosis and management of a febrile child with valvulitis and significant haemodynamic disturbance.

The differential diagnosis between the two most likely aetiological factors was made difficult by the previous administration of antibiotics and by the absence of laboratory evidence of a recent streptococcal infection. Although our patient had one major and two minor criteria for the diagnosis of acute rheumatic fever, there was no evidence of a preceding streptococcal infection as required in the 1965 revision of the Jones's criteria, ${ }^{1}$ until 8 weeks after the onset of symptoms.

It was clear that cardiac surgery was required but our dilemma was that we were unable to exclude subacute bacterial endocarditis and indeed, in view of the comparative rarity of acute rheumatic carditis of this severity, we felt that an infective endocarditis was the more probable cause. We therefore considered it advisable to delay surgery until we could ensure that sufficient antibiotic treatment had been administered to render this intervention less hazardous. Although our preoperative diagnosis was shown to be wrong, we believe that faced with the same situation we would still pursue the same course of management.

In accordance with previous reports, ${ }^{2-4}$ this case shows that surgical intervention in the acute phase of rheumatic fever is safe and beneficial; in the critically ill patient it may even be life-saving.

We thank Mr J Cleland, senior consultant cardiac surgeon, Royal Victoria Hospital, Belfast, for help in the management of this case.

\section{References}

1 Stollerman G J H, Markowitz M, Taranta A, Wannamaker L W, Whittemore R. Jones' criteria (revised) for guidance in the diagnosis of rheumatic fever. Circulation 1965; 32: 664-8.

2 Timmis H H, Hardy J D, Watson D G, Blake T M. Mitral valve replacement in a child during acute rheumatic carditis. Ann Surg 1966; 164: 1034-40.

3 Strauss A W, Goldring D, Kissane J, et al. Valve replacement in acute rheumatic heart disease. J Thorac Cardiovasc Surg 1974; 67: 659-70.

4 Khan A, Chi S, Gonzalez-Lavin L. Aortic valve replacement during acute rheumatic fever. Br Heart J 1978; 40: 817-9.

Correspondence to Dr Patricia Morton, Belfast City Hospital, Lisburn Road, Belfast BT9 7AB.

Received 28 June 1982

\title{
Infantile multicystic encephalomalacia after maternal bee sting anaphylaxis during pregnancy
}

\author{
C ERASMUS, W BLACKWOOD, AND J WILSON
}

The Hospital for Sick Children, London, Queen Mary's Hospital for Children, Carshalton, Surrey, and Hurstwood Park Hospital, Haywards Heath, Sussex

SUMMARY We report a case of infantile multicystic encephalomalacia after maternal bee sting anaphylaxis in the 30th week of pregnancy. The clinical features and findings at necropsy are described, and it is suggested that these are the result of severe fetal hypoxia secondary to maternal hypotension.

In multicystic encephalomalacia, cerebral necrosis and cavitation occur after cerebral insult. The tendency for the immature brain to undergo cavitation is a function of the timing ${ }^{1}$ and nature of the insult. ${ }^{1-3}$ It is especially likely to occur between the 6th month of gestation and the early postnatal period. ${ }^{1}$ Severe asphyxia or circulatory disturbances are of major aetiological importance. ${ }^{1-4}$
Very few neuropathological studies have been reported in which a specific antenatal insult could be identified. ${ }^{56}$

\section{Case report}

The pregnancy of a healthy non-consanguineous 31-year-old primigravida progressed normally, under regular obstetric supervision until the 30th week, when she was stung by a bee. She developed severe anaphylaxis and was unconscious for about 2 hours before admission to an intensive care unit. On examination there, she was drowsy and had facial oedema. Her blood pressure was $60 / 0 \mathrm{mmHg}$. She responded rapidly to intravenous hydrocortisone acetate and after 3 hours her blood pressure was 
$120 / 80 \mathrm{mmHg}$ and her condition was clinically much improved. The fetal heart rate then was $144 /$ minute and regular. She was discharged 24 hours after admission, her condition being satisfactory. Fetal movements ceased for 4 days subsequently and the fetal heart was inaudible.

An ultrasound scan, performed 6 days after the anaphylaxis, showed an increased fetal biparietal diameter compared with the value predicted from a 16-week measurement (Fig. 1). A large cystic mass occupying one-third of the fetal abdominal area could be seen and was thought to represent a grossly distended bladder. The biparietal diameter was still increased on ultrasound scan 10 days later, although $2.5 \mathrm{~mm}$ smaller than previously. The cystic mass was not seen, but polyhydramnios was demonstrated; this was a new finding.

Spontaneous onset of labour occurred at the 35th week, 34 days after the bee sting. The liquor was meconium stained. A limp and cyanosed $2 \cdot 18 \mathrm{~kg}$ girl was delivered normally after labour lasting 1.3 hours. Apgar scores were 4 at one minute and 7 at five

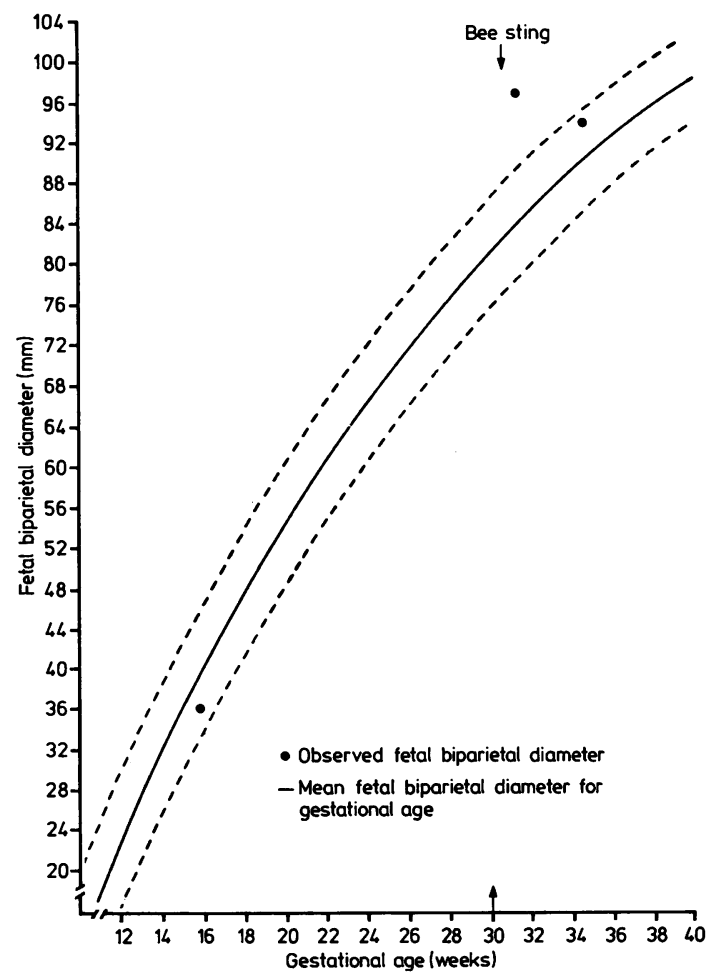

Fig. 1 Ultrasound measurements of fetal biparietal diameter showing the mean for gestational age, $95 \%$ confidence limits, and observed measurements. Note the increase, with subsequent reduction after the bee sting. minutes. The placenta weighed $490 \mathrm{~g}$ and was gritty. The infant's gestational age was estimated at 36 weeks. Her head circumference was $32 \mathrm{~cm}$ with marked over-riding of sutures. She had bilateral genu recurvatum and dislocated hips. Her face was immobile and apart from an occasional highpitched cry, she was quiet, unresponsive, with no spontaneous movements. Her temperature control was poor. There were severe feeding difficulties. Moro and grasp reflexes were present, but she had no gag, sucking, or swallowing reflexes. Her fundi were normal and eye movements were full. The upper limbs were hypotonic at birth, but became hypertonic within 4 weeks. The lower limbs were hypertonic at birth. At age 4 weeks all tendon reflexes were exaggerated.

A full biochemical and infection screen was normal.

At 4 weeks an electroencephalogram showed poverty of normal activity over both hemispheres and computerised tomography showed marked cerebral atrophy with increased density in the posterior thalami and cystic change in the parietal subcortical substance.

The head circumference remained $32 \mathrm{~cm}$, the sutures overlapping. The infant made no progress and she died at 64 days.

\section{Necropsy}

The brain was symmetrically small and firm. Bilateral cystic cavitation affected the white matter deep to the insulae and the superomedial aspect of the posterior frontal and parietal lobes (Fig. 2). The cortex overlying the cavities was thin, with microscopic loss of neurons and replacement gliosis. Smooth-walled cavities replaced most of each globus pallidus and putamen. Widespread cell loss and gliosis in the thalami and severe hippocampal sclerosis was present, as well as boundary zone

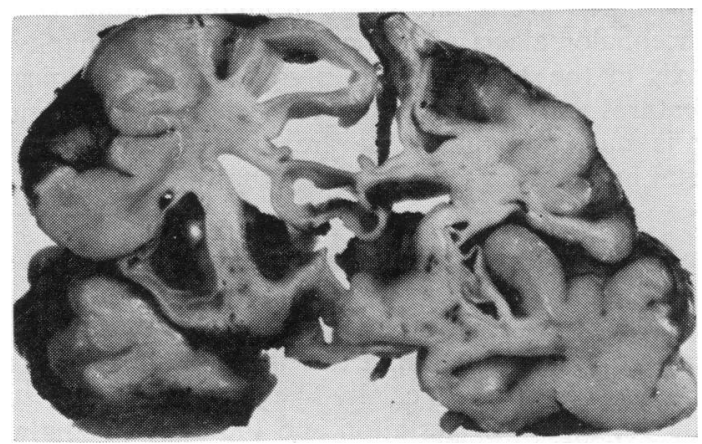

Fig. 2 Coronal section of brain showing atrophy, cavitation, and germinal haemorrhage. 
sclerosis of the cerebellum. In each paraventricular germinal zone there was old haemorrhage with surrounding gliosis. The brain stem was much reduced in size. Most noticeable was nerve cell loss with gliosis in the reticular zones of the midbrain, pons and medulla, the colliculi, substantia nigra, red and inferior olivary nuclei. A lesser degree of neuronal loss occurred from the 3rd, 4th, and 7th cranial nerve nuclei. The cuneate, gracile, and other cranial nerve nuclei appeared normal. The internal arcuate and medial lemniscal fibres were reduced in number and poorly myelinated. The pyramidal tracts were reduced in size and unmyelinated.

\section{Discussion}

Multicystic encephalomalacia with striatal and brain stem damage is seen after severe hypoxicischaemic insult to the brain, ${ }^{4-8}$ and has been demonstrated experimentally in monkey fetuses. ${ }^{2} 3$ The extent and distribution of injury to brain structures are determined by their inherent vulnerability, ${ }^{1-3}$ the degree and duration of asphyxia, ${ }^{23}$ and the presence of acidosis. ${ }^{3}$ The structures' susceptibility to injury corresponds to the rank order of their intrinsic metabolic rates and blood volume flow. ${ }^{1-3}$

There is a close correlation between maternal mean aortic pressure, placental perfusion, and fetal oxygen partial pressure. ${ }^{2}$ In monkey fetuses, asphyxia leads to diminished ventricular contractility, myocardial injury, decreased cardiac output, and hypotension. ${ }^{2}$ Impairment of cerebral blood flow and loss of cerebral vascular autoregulation occur. ${ }^{1}$

A hypoxic fetal brain becomes severely oedematous, further compromising cerebral perfusion, thus evoking a vicious cycle. The biochemical derangement after ischaemia-hypoxia ${ }^{1}$ and failure to re-establish cerebral microvascular perfusion (noreflow phenomena) 12 lead to patchy cerebral infarction.

In the case reported here it is probable that the fetus was hypoxic during a period of prolonged maternal hypotension. This resulted in severe fetal cerebral oedema as shown by a striking increase in fetal biparietal diameter with subsequent reduction (Fig. 1). The distended fetal bladder, seen on ultrasound scan, may have been due to urinary retention secondary to hypoxic spinal cord damage. Unfortunately only a small rostral segment of cord was available for examination. Histological evidence of spinal cord injury after intrauterine hypoxia has been described. ${ }^{2} 8$ Likewise, the absence of fetal movements for 4 days after the bee sting was highly suggestive of neuraxial insult, the legacy of which could be seen in the limb deformities. The inability to swallow, so obvious after birth, may have contributed to polyhydraminos in utero.

There have been many reports of multicystic encephalomalacia, two of which deserve mention..$^{56}$ In the first, ${ }^{5}$ intravenous iron caused severe maternal anaphylaxis in the 34th week of pregnancy. A girl who survived for 4 months, was delivered 4 days later, with clinical and neuropathological findings very similar to our case. In the other case, ${ }^{6}$ a mother attempted to commit suicide by inhalation of carbon monoxide in the 35th week of pregnancy. Her son was delivered without signs of birth asphyxia 5 weeks later and survived for 5 days. The clinical and pathological findings paralleled our own case.

Although in our patient there was some evidence of fetal distress at birth, with meconium staining of liquor, it is unlikely that this caused the severe changes seen at necropsy, except for the germinal haemorrhages which may have occurred perinatally. We believe that the condition can be explained entirely by fetal hypoxia secondary to severe maternal hypotension after bee sting anaphylaxis.

We thank Dr F B Briggs, Dr G W Hatcher, Dr A Frost, and Dr Wemyss-Gorman for helpful information; R I K Elliot for the specimen of brain; Mrs S Karle, ultrasonographer, for assistance; Miss Susan Langridge for typing the manuscript.

\section{References}

1 Volpe J J. Neurology of the newborn. Vol. XXII. Major Problems in Clinical Pediatrics. Philadelphia: Saunders, 1981: 141-238.

2 Myers R E. Two patterns of perinatal brain damage and their conditions of occurrence. Am J Obstet Gynecol 1972; 112: 246-76.

3 Myers R E. Four patterns of perinatal brain damage and their conditions of occurrence in primates. Adv Neurol 1975; 10: 223-34.

4 Manterola A, Towbin A, Yakovlev P I. Cerebral infarction in the human fetus near term. $J$ Neuropathol Exp Neurol 1966; 25 : 479-88.

5 Sharpe O, Hall E G. Renal impairment, hypertension, and encephalomalacia in an infant surviving severe intrauterine anoxia. Proc Roy Soc Med 1953; 46: 1063-5.

- Neuburger F. Fall einer intrauterinen Hirnschädigung nach einer Leuchtgasvergiftgung der Mutter. Beitr Gerichtl Med 1935; 13: 85-95.

7 Aicardi J, Goutières F, de Verbois A H. Multicystic encephalomalacia of infants and its relation to abnormal gestation and hydranencephaly. J Neurol Sci 1972; 15: 357-73.

8 Smith J F, Rodeck C. Multiple cystic and focal encephalomalacia in infancy and childhood with brain stem damage. J Neurol Sci 1975; 25: 377-88.

Correspondence to Dr Celia Erasmus, H F Verwoerd Hospital, Private Bag X 169, Pretoria, Republic of South Africa.

Received 1 June 1982 